\title{
The Early Phases of Enterprise Knowledge Modelling: Practices and Experiences from Scaffolding and Scoping
}

\author{
Kurt Sandkuhl ${ }^{1}$ and Frank Lillehagen ${ }^{2}$ \\ ${ }^{1}$ School of Engineering at Jönköping University, \\ P.O. Box 1026, 55111 Jönköping, Sweden \\ Kurt.Sandkuhl@jth.hj.se \\ ${ }^{2}$ Active Knowledge Modeling AS, \\ P.O. Box 376, 1326 Lysaker, Norway \\ f.lillehagen@akmodeling.com
}

\begin{abstract}
Enterprise modelling concepts, methods or technologies have been found useful for a variety of application areas and purposes. The experience report presented in this paper is from the area of enterprise knowledge modelling with an application case in automotive supplier industries. The purpose of the overall modelling process is to create an active knowledge model. The scope of the paper are the early phases of the enterprise knowledge modelling process, called scaffolding phase and scoping phase. An important observation is the need for continuous and intertwined development of metamodel, model, modelling process and modelling team.
\end{abstract}

Keywords: Enterprise knowledge modelling, practice report, active knowledge modelling.

\section{Introduction}

During the last 20 years, enterprise modelling concepts, methods or technologies have been found useful for a variety of application areas and purposes, like understanding and improving the business processes in an organization, capturing requirements in software system development, visualizing document and information flow, preparing strategic decisions in IT governance, analyzing fraud risks, evaluating business value of IT investments, orchestrating the project validation, and many more.

As a consequence, researchers and practitioners from many different disciplines have been contributing to development of enterprise modelling practices, including information system development, software engineering, enterprise engineering or organizational development.

The experience report presented in this paper is from the area of enterprise knowledge modelling with an application case in automotive supplier industries. The objective of the research work performed was to develop and evaluate a model-based collaboration infrastructure for use in distributed product design. In this context, modelling of the relevant part of the application case was initiated. The purpose of the modelling process was to create an active knowledge model, i.e. an enterprise 
knowledge model, which supports execution of work tasks and is adaptable to the user's local demands (cf. section 2.2). The scope of the paper is the early phases of the enterprise knowledge modelling process, called scaffolding phase and scoping phase. These phases were selected for the experience report as they resulted in experiences and work practices of potential interest for enterprise modelling in general.

The paper is structured into four more chapters: Chapter 2 will summarize the background for our work including the industrial case considered, the area of active knowledge modelling and the modelling approach used. Chapter 3 will present the practice of scaffolding and scoping. Chapter 4 discusses limits and potentials of the scaffolding practices. Chapter 5 summarizes the achievements.

\section{Background}

The experience report presented in this paper is based on an industrial case, which is introduced in section 2.1. Furthermore, a short introduction to the specifics of active knowledge modelling will be given (section 2.2) and the modelling approach used will be presented (section 2.3).

\subsection{Industrial Case}

The experiences presented are based on work in the EU-FP6 project MAPPER ${ }^{1}$ (Model-adapted Process and Product Engineering). MAPPER had a runtime from autumn 2005 to spring 2008 and aimed at enabling fast and flexible manufacturing in networked enterprises by providing methodology, infrastructure and reusable services for participative engineering.

The industrial case considered in this paper was a use case in the MAPPER project and focuses on distributed product development and multi-project lifecycles in a networked organization with different subsidiaries of an automotive supplier. The main partner is the business area "interior" of a first tier automotive supplier with the main product development sites in Scandinavia. The interior area mainly includes seat comfort products like seat heater, seat ventilation, climate control, lumber support and head restraint. During the MAPPER project, the focus was on the advanced engineering unit, where product development tasks are concentrating on predevelopment of new concepts and new materials.

Development of products includes elicitation of system requirements based on customer requirements, development of functional, design of logical and technical architecture, co-design of material, electrical and mechanical components, integration testing and production planning including production logistics, floor planning and product line planning. The process is geographically distributed involving engineers and specialists at several locations of the automotive supplier and sub-supplier for specific tasks. A large percentage of seat comfort components can be considered as product families, i.e. various versions of the components exist and have to be maintained and further developed for different product models and different customers. In this context, flexible product development in networks with changing partners on customer and sub-supplier side is of crucial importance.

\footnotetext{
${ }^{1}$ See [1] for more information about MAPPER.
} 
The purpose of the enterprise knowledge modelling in this use case was to capture the relevant product knowledge and process knowledge required for supporting collaborative engineering at different sites of the automotive supplier. The knowledge model was expected to contribute to solving a number of challenges:

- To support fast integration of geographically distributed collaboration partners

- To enable flexible development processes, combining pre-defined processes for coordinated development tasks and ad-hoc process changes.

- To coordinate a large number of parallel product development activities

\subsection{Enterprise Knowledge Modelling and Active Knowledge Models}

Enterprise knowledge modelling is applying and extending approaches, concepts and technologies from enterprise modelling and enterprise architecture for knowledge representation and knowledge-based solutions. In general terms, enterprise modelling is addressing the systematic analysis and modelling of processes, organization structures, products structures, IT-systems or any other perspective relevant for the modelling purpose. Enterprise models can be applied for various reasons, like visualization of current processes and structures in an enterprise, process improvement and optimization, introduction of new IT solutions or analysis purposes. [9] provides a detailed account of enterprise modelling and integration approaches including reference models.

The field of enterprise architectures received a growing attention during the last years in the context of IT-governance or corporate governance. Main intention is to visualize the architecture of IT-application and infrastructure in an enterprise including the supported processes or organization units. Such enterprise architecture models are closely related to enterprise models, as they to a significant extent have to cover the same aspects (e.g. processes and organization structures). However, enterprise architectures focus on providing a basis for analyzing current IT architectures in enterprises and providing a basis for (strategic) planning of future developments. [10] discusses in particular the connection between enterprise architecture and IT governance.

Enterprise knowledge modelling combines and extends approaches and techniques from enterprise modelling and enterprise architectures. The knowledge needed for performing a certain task in an enterprise or for acting in a certain role has to include the context of the individual, which requires including all relevant perspectives in the same model. Using the knowledge is applying different reflective views on the knowledge model. Enterprise knowledge modelling aims at capturing reusable knowledge of processes and products in knowledge architectures supporting work execution [2]. These architectures form the basis for model-based solutions, which often are represented as active knowledge models [3]. [4] identifies characteristics of active models vs. passive models and emphasize that "the model must be dynamic, users must be supported in changing the model to fit their local reality, enabling tailoring of the system's behaviour".

In MAPPER, these active knowledge models included the POPS* perspectives [5]:

- the process perspective $(\mathrm{P})$ captures the work processes and tasks in the networked enterprise, 
- the organization perspective $(\mathrm{O})$ includes all roles involved in the processes and their skills and competence profiles,

- the product perspective (P) focuses on components, configuration possibilities and dependencies of the product under consideration,

- the systems perspective (S) includes the IT systems supporting work processes and product development,

- further perspectives $(*)$ depend on the requirements of the enterprise under consideration and can include business objectives, customer requirements regarding the products or key success factors.

These perspectives are mutually reflective, i.e. each perspective influences content and meaning of the other perspectives, which is captured in relationships and dependencies between the elements of the perspectives. The active knowledge model developed in MAPPER can be executed in the MAPPER infrastructure, which for example includes a basic work flow engine and model-configured role-specific work places for capturing product knowledge.

\subsection{Enterprise Knowledge Modelling with C3S3P}

Enterprise knowledge modelling in the industrial case was performed according to the $\mathrm{C} 3 \mathrm{~S} 3 \mathrm{P}$ methodology. C3S3P is based on work in several EU projects from the area of networked and extended enterprises. An extended enterprise is a dynamic networked organization, which is created ad-hoc to reach a certain objective using the resources of the participating cooperating enterprises. In order to support solutions development for extended enterprises, the EXTERNAL project developed a methodology for extended enterprise modelling [6], which initially was named SGAMSIDOER. This methodology was further developed towards a complete customer delivery process denoted C3S3P, which was used in the ATHENA ${ }^{2}$ and MAPPER projects.

C3S3P distinguishes between seven phases called Concept study, Scaffolding, Scoping, Solution modelling, Platform integration, Piloting in real projects and Performance monitoring and management. The $\mathrm{C} 3 \mathrm{~S} 3 \mathrm{P}$ phases roughly include the following:

- Concept Study: pre-studies are performed to investigate whether EM is a suitable and accepted way of developing executable solutions for the networked enterprise

- Scaffolding ${ }^{3}$ aims at creating shared knowledge and understanding among the participants of the project about the scope and challenges of the project.

- Scoping: creation of models supporting the networked enterprise for a defined scope including all relevant dimensions required, like process, product, organization or IT-systems

- Solutions Modelling: refining the scoping model by integration personnel, product structures, document templates and IT systems required for using the enterprise model in an actual project

\footnotetext{
${ }^{2}$ http://www.athena-ip.org/

${ }^{3}$ The term scaffolding indicates the intention of this phase to create a firm structure supporting the development of a solution without making this structure a part of the solution - like in construction projects where the scaffold supports the construction of a building.
} 
- Platform Configuration: configure the solution models for use in the networked or extended enterprise by connecting the enterprise model to the platform used (see [1] for details on the MAPPER platform)

- Platform Delivery: encompasses the roll-out of model-configured solutions

- Performance Improvement by capturing indicators for process and product quality and using adequate management instruments.

The work performed in MAPPER included two cycles of using C3S3P. The first cycle focused on capturing organizational knowledge and best practices for networked manufacturing enterprises. The second cycle focused on integration of product knowledge into the best practices. The practices presented in the following chapter are primarily taken from the first cycle and focus on scaffolding and scoping. The scaffolding phase of the second C3S3P cycle was considerably shorter than in the first cycle, as a lot of shared understanding of the problem domain already had been created.

\section{Practice of Scaffolding and Scoping}

The objective of this chapter is describe the way of working during scaffolding and scoping, including practices applied, some illustrative examples of the models produced and an overall structure of the early phases of this enterprise knowledge modelling process.

\subsection{Scaffolding Phase}

Before starting the scaffolding phase, a concept study was performed, which aimed at creating a shared understanding of the task at hand, the product design terminology used, and the organizational context at the automotive supplier. During this study, the automotive supplier basically gave a detailed introduction into aspects like what are seat comfort products and components, and how are they developed and manufactured. This introduction included visits at the engineering labs, manufacturing plant and test facilities.

\section{Preparation Steps}

At the beginning of the scaffolding phase, a number of preparation steps had to be taken, which were contributing to a joint understanding of visual knowledge modelling and a prerequisite for the later phases. After having identified all use case team members from the involved MAPPER partners, the team members were offered an introduction and basic training in the METIS $^{4}$ tool, which was selected as modelling tool. Furthermore, an agreement was made to start with scaffolding workshops involving all team members and to locate these workshops at the automotive supplier's premises, in order to have quick access to stakeholders and material, which could support modelling. An initial distribution of roles to persons was made, which included the following roles for the modelling process:

4 The METIS tool was recently renamed into Troux Architect. For tool information see www.troux.com 
- Manager: the owner of the use case who is responsible for establishing the use case at the use case partner, assigning the right personnel resources, arranging meetings, etc.

- Planner: the person responsible for proposing the way of working and establishing a consensus between all partners, coordinating the different tasks, moderating the meetings, etc.

- Modelling expert: provides expert knowledge in modelling process, methods and tools to the use case team

- Facilitator: is experienced in using the selected modelling process and tool and facilitates model construction and capturing of knowledge in the models

- Coach: supports the modelling process and model development by coaching the modellers

- Modeller: develop the enterprise models in the selected tool during the modelling process

- Domain expert: provide knowledge about the domain under consideration, which is basis for modelling

\section{Modelling}

Based on the above preparations, an iterative process started, which at the end resulted in 3 major versions of the scaffolding model. The first actual modelling step

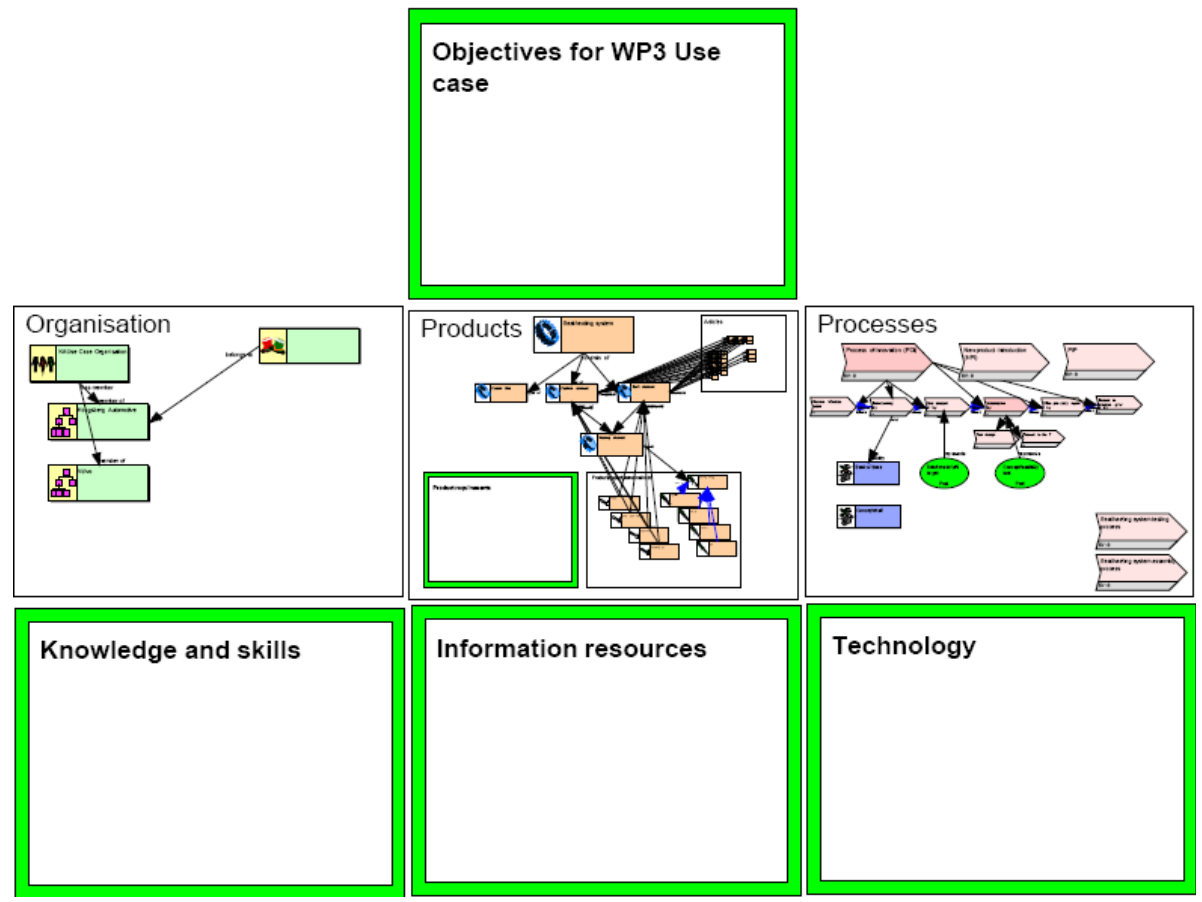

Fig. 1. The first scaffolding model basically identified the required modelling perspectives. Most of the boxes, which are called containers for the sub-models, were empty in this phase. 

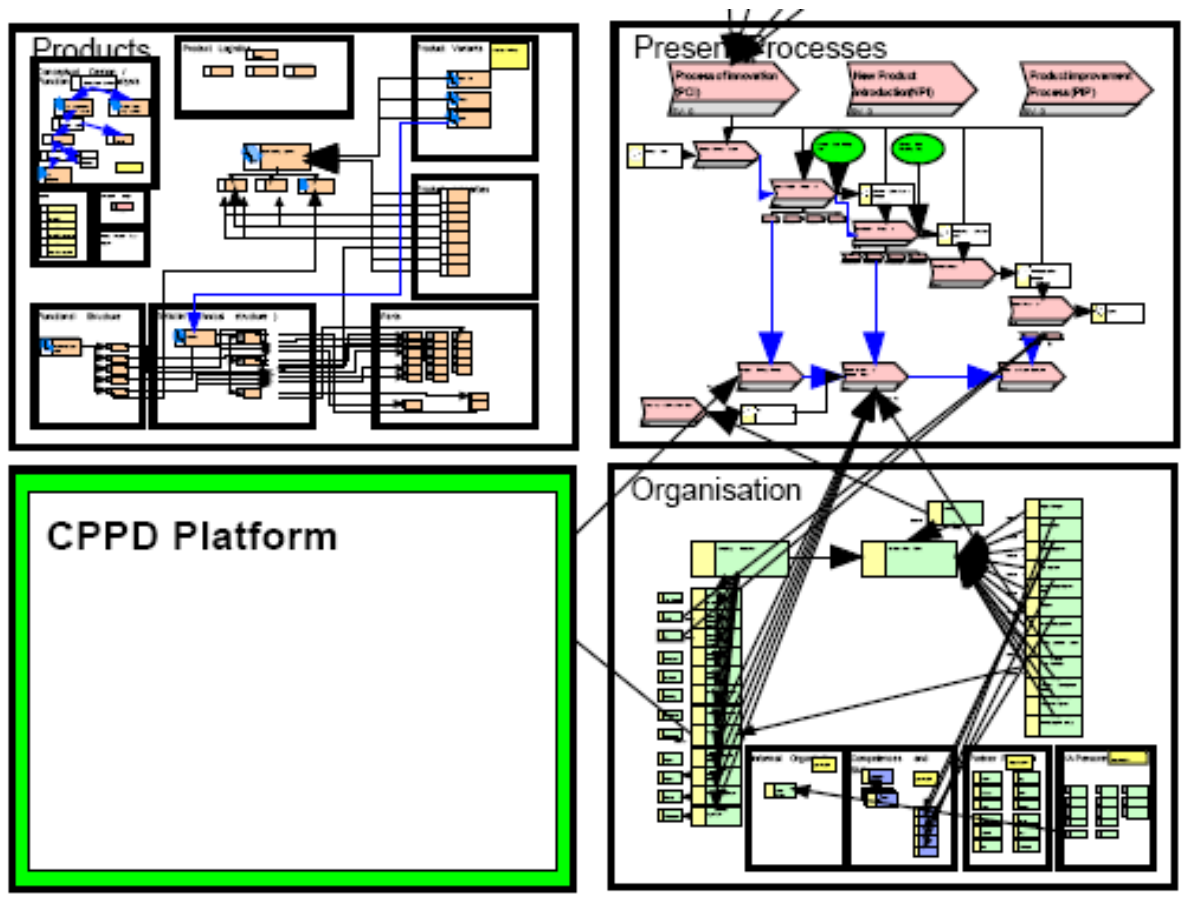

Fig. 2. The 3rd scaffolding model version included more details for each perspective. The figure shows the organization, product and process containers.

in the first iteration was to clearly define the purpose of the model to be developed. Within the scaffolding phase, the purpose was to model the current situation in the use case subject as seen by the different stakeholders from the automotive supplier (R\&D manager, engineers with different specialisations, purchaser, customer responsible, etc.) in order to create a joint understanding in the complete use case team. Starting from the POPS* approach, the perspectives were identified that were considered relevant for the modelling purpose. The initial POPS* perspectives Process, Organisation, Product and Systems were during this step supplemented with other perspectives like Objectives, Technical Approaches or Skills. The definition of additional perspectives had to be repeated in all iterations.

The modelling work performed in joint workshops with all use case team members consisted of a combination of presentations with regards to the current situation in the use case subject, discussions for clarifying concepts and creating a joint understanding, and model creation and editing. The modelling work usually was structured by differentiating between sessions focusing on single perspectives, like process or organisation, and sessions aiming at inter-perspective relationships. This way of working turned out to create the necessary balancing between capturing very detailed aspects within the perspectives and more general aspects.

The modelling workshops usually were 2 or even 3 day events. Between two workshops, the coach and modelling expert checked the jointly developed models and details like textual descriptions were added. At the beginning of each consecutive 


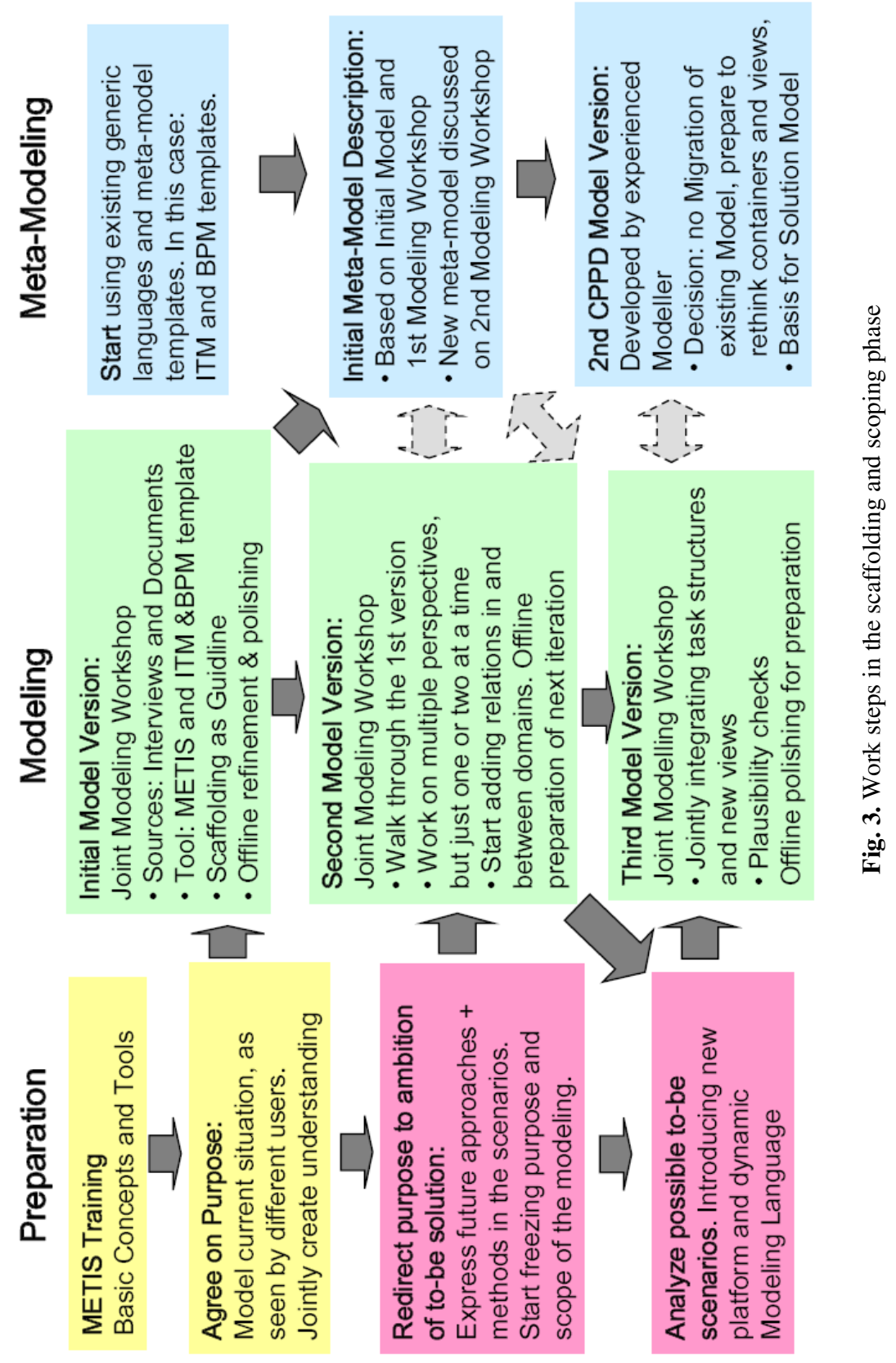


modelling workshop, a walk through the current model version was the first step in order to make sure that all participants had the same understanding of the status and in order to verify the modifications made between the workshops. After the $3^{\text {rd }}$ model iteration in the scaffolding phase, the scaffolding model was considered detailed enough and sufficiently complete.

\section{Meta Modelling}

Besides the actual modelling, development of a suitable meta-model for the purposes of the use case was another important subject. During the scaffolding phase, the work was based on the $\mathrm{BPM}^{5}$ and $\mathrm{ITM}^{6}$ meta-models provided by Troux, which are available as templates for the METIS tool. Experience from the modelling workshop showed that these two templates and their respective modelling elements covered a large part of the needs. Missing model elements were mainly identified for the "Product" perspective, as different conceptual views on a product (like geometric, electrical, material or cost view) have to be supported and interconnected in the same model. In order to provide suitable elements, work on a new meta-model was started, which should integrate the required elements of BPM and ITM and add specific constructs for the product view. This meta-modelling activity was initiated by Troux and led to an initial meta-model proposal for Collaborative Product and Process Development (CPPD). After an initial meta-model version, work on CPPD was performed in parallel with development of the scaffolding model and continued even during scoping.

Fig. 3 illustrates the activities in the scaffolding phase, which can be divided into preparation work, modelling and meta-modelling.

\subsection{Scoping Phase}

The scoping phase used the same tools and roles as the scaffolding phase, but had a different purpose: Focus in the scoping phase was on developing initial versions of solution models that specified the intended future way of working in the use case subject, i.e. the future product development process at the automotive supplier's business area seat comfort. Furthermore, the solution models should be executable in the MAPPER infrastructure, i.e. all modelling perspectives had to be defined on such a level of detail that they contained all model elements required for execution. In comparison to the scaffolding model, the solution model had to fulfil higher demands with respect to completeness and consistency, e.g. the complete process flow or set of required tasks within a process had to be modelled and not just the essential ones illustrating the way of working. Furthermore, much more technical details had to be included, e.g. all collaboration services needed, internal IT-systems and information sources required to support a task had to be specified including their technical interfaces.

Due to the requirements regarding completeness, consistency and level of detail, the way of modelling had to be revised. Jointly working on METIS models in workshops and creating all model elements jointly was no longer appropriate. Instead, we decided to first create textual scenario descriptions for all relevant tasks. These

\footnotetext{
${ }^{5} \mathrm{BPM}=$ Business Process Modelling.

${ }^{6}$ ITM = Information Technology Management.
} 
textual descriptions included information about the intended way of working, involved roles, and tools or documents used. They also contained statements explicitly identifying requirements. Based on each scenario description, a model was developed by the coach and modelling expert, which was afterwards presented and discussed with the team members from the automotive supplier. The joint modelling workshops performed during the scoping phase aimed at presenting the current modelling status, and at discussing questions like how to integrate collaboration support on base of the solution model, how to integrate methodology support into the solution model, how model development and meta-model development correspond or how the future distribution of work would look like. Thus, the modelling workshops, which always started with a walk through the current model status, still formed an essential part of the process.

The work during the scoping phase was structured by dividing the design process into 9 main tasks and grouping these 9 tasks into 3 pilot installations. The tasks for the first pilot were given priority and developed first. As a consequence, models for these tasks existed early in the process already in a $2^{\text {nd }}$ or $3^{\text {rd }}$ version, while some of the tasks for pilot 2 and 3 were available only in a first iteration.

The solution modelling phase, i.e. refining the scoping model for use in an actual project, is not in the scope of this paper. Selected aspects of solution modelling as process and an example for the solution model are discussed in [1].

\section{Experiences}

Many experiences and practices regarding scaffolding and scoping are already included in section 3. This section adds experiences regarding stakeholder participation and the two cycles of C3S3P performed.

As indicated above, participation of stakeholders from different departments of the automotive supplier and from different MAPPER partners has to be considered a key success factor for creating enterprise knowledge models suitable for use in everyday practice. However, we experienced that different levels of participation were adequate for the different modelling phases (see section 2.2 for a description of the phases).

In the initial phase, scaffolding, nearly all stakeholders were participating all the time during the model development process. This included presentations from and discussions with the automotive supplier about the application domain and the use case subject, discussions and joint decisions about perspectives to be included in the model, consideration about the meta-model, joint creation and editing of the models with METIS and textual descriptions of the model elements. In terms of the produced size of models, these model sessions might not have been very productive. But in terms of sharing knowledge and creating a joint understanding of both, the use case subject and the nature and process of modelling, the joint modelling sessions with all stakeholders were extremely valuable. They created a joint ownership of the result. Only between the modelling sessions, there was a "non-participatory" work step, which concerned the consolidation of the model and the meta-model. Introducing the results of this work step to all stakeholders at the beginning of the next modelling session by walking through the model was very useful. This contributed to getting everybody onto the same level of information and supported double-checking of understandability and correctness of the model. 
During the second phase, scoping, the way of working was changed and the level participation reduced: before developing METIS models, we developed textual scenario descriptions for the task under consideration. The scenario descriptions were developed with participation of all stakeholders, but the development of models based on the scenario description was done "in private" by the modelling expert. Main reason for this change was the required level of detail of the models. As the objective was to provide executable models, a lot of detailed and partly technical information had to be included. Joint editing of such models was not only perceived very time consuming but also overloading some stakeholders with technical details considered not relevant for them. However, the models produced by the expert were presented to the other stakeholders for validation purposes and in order not to loose the joint ownership of the results.

The work performed in MAPPER included two cycles of using C3S3P. The first cycle focused on capturing organizational knowledge and best practices for networked manufacturing enterprises. The second cycle focused on integration of product knowledge into the best practices. The scaffolding phase of the second C3S3P cycle were considerably shorter than in the first cycle, as a lot of shared understanding of the problem domain already had been created. However, there still was the need to explore the principal design solution, configurable components and parameters of the product area under consideration. The scoping phase to a large extent consisted of identifying the required configurable workplaces, which were created during the solution modelling phase. Platform integration and piloting in real-world projects were not clearly separated due to the tight project schedule. Creation of workplaces for engineers at the use case partner in running projects was the main aim. The performance monitoring phase was not yet performed (see [8] for a more detailed discussion).

For the industrial partner participating in the described case, developing enterprise knowledge models was a new experience. Before the beginning of MAPPER, modelbasing for them primarily meant the use of simulation models or geometric models in product design. During the second C3S3P cycle it became very clear that the future use of knowledge models and modelling approaches at the use case partner would depend on establishing changed work practices and on a better understanding in larger parts of the company what potentials and benefits knowledge modelling can contribute. As a consequence, work on social practice design [14] was initiated aiming at creating a knowledge modelling culture.

\section{Related Work}

In contrast to the wealth of publications discussing concepts, techniques, tools, methodologies or selected aspects of enterprise modelling, there are relatively few experience reports or best practices published. Most relevant for a related work discussion would be work using the same methodology (C3S3P). But this would limit the discussion to only a few publications, which all were already mentioned earlier in this article $[6,7,8]$. Thus, this section will also discuss experiences from neighbouring areas. 
[11] state quite similar goals and perspectives in their work on enterprise modelling, which is tightly related to the $\mathrm{TOVE}^{7}$ project. They aim at "a computational representation of structure, activities, processes, information, people, behaviour, goals, and constraints of a business, government, or other enterprise". Both, the objective of a formal, computational model and the different perspectives captured in such models are very close to the objective of the enterprise knowledge modelling performed in our case and the perspectives of POPS*. However, TOVE puts much more focus on functional completeness, efficiency, minimality or generality, using an ontological approach and representation. From our experience, we would support the importance of these quality criteria, but emphasize the need for a visualization of the enterprise model and all relations between the different perspectives that is easily understood and consistently applied and interpreted in the enterprise. Visual models as used for scaffolding and scoping in MAPPER seem to have an advantage in supporting this goal as compared to ontological representations.

Our experiences regarding role distribution and user participation are supported by other researchers. [12], for example, in their experience report using the EKD enterprise modelling method emphasize the importance of a clearly stated mission (in our case, the agreement between the stakeholders about the purpose of the modelling phase), of team composition and in particular the role of the facilitator, and of adequate tool support. Furthermore, they emphasize the need for combining modelling language and adequate modelling process, which fits to our view that meta-model and modelling process should be adjusted before and during the modelling work.

The scaffolding phase spends considerable time and efforts on identifying the needed perspectives of an enterprise model and on understanding the situation under consideration. Only after several scaffolding iterations, we moved on to the scoping phase, i.e. toward preparing solutions for the identified problems and objectives. The advantage of this approach is a tight integration between the different perspectives from the very beginning, i.e. all perspectives of the scaffolding model were elaborated in sufficient detail and thoroughly related to the other perspectives. We experienced this as very useful for the scoping phase, as it helped to raise awareness for important commonalities or dependencies between perspectives and objects, and for essential relationships within the considered case. This experience we share with [13], even though they address in their work a completely different application field. Their work on ERP systems showed that expressing organizational needs in goal-strategy terms by using a visualization easily understood by the enterprise helps to take a more holistic perspective. The "map" applied in their work includes so diverse perspectives as requirements, system parameters, strategies, or functionality. The experiences reported are that this was very useful in focusing on dependencies and strategically important questions.

\section{Summary}

The paper presented practices and experiences from the early phases of enterprise knowledge modelling at an automotive supplier. The objective of the model development

${ }^{7}$ TOVE $=$ Toronto Virtual Enterprise. 
was to support collaborative product design in a networked manufacturing enterprise. The modelling approach used was C3S3P, the scope of the paper included the scaffolding and the scoping phases.

One of the most important observations from the modelling case described is the continuous and intertwined development of several perspectives:

- The meta-model, which evolved from an off-the-shelf template to a specialized meta-model for collaborative product and process development,

- The model as such with the different phases described in chapter 3. It should be noticed that the models resulting from scaffolding and scoping could be considered as both, artefacts on their own rights and transient results between two model phases

- The modelling process was guided by the C3S3P phases, which offers a high degree of freedom in terms of how to implement the phases. The modelling process as such therefore was subject of continuous adaptation based on the progress and evolving necessities in the modelling sessions performed.

- The modelling team and partly the role distribution in the team changed in course of the work, as different domain experts or specialist for knowledge modelling of particular perspectives had to integrated

The recommendation resulting from this observation is to establish during enterprise knowledge modelling, in particular if C3S3P is applied, a methodology adaption task focusing on identifying and tackling the needs for adjustment in the above discussed perspectives. Investigating this evolutionary process and identifying key success factors based on wider empirical grounding and existing work in method development would be an interesting future line of work.

Furthermore, the differences, advantages and disadvantages of $\mathrm{C} 3 \mathrm{~S} 3 \mathrm{P}$ in comparison to other enterprise modelling methodologies, like EKD [15], should be investigated and exposed. For the case discussed in this paper, C3S3P was predetermined as methodology. Although the experiences with C3S3P were positive, a comparison to other approaches would be valuable.

\section{Acknowledgements}

The authors wish to thank the anonymous reviewers of the paper for their valuable comments.

The paper is to a large part based on research carried out in the EU project FP6IST-NMP 016527 MAPPER "Model-adapted Product and Process Engineering". The authors wish to thank their co-workers when developing the MAPPER enterprise knowledge models. Furthermore, the work was supported by the Swedish Knowledge Foundation with grant \#2005-0252 (project infoFLOW).

\section{References}

1. Johnsen, S., et al.: Model-based adaptive Product and Process Engineering. In: Rabe, M., Mihok, P. (eds.) Ambient Intelligence Technologies for the Product Lifecycle: Results and Perspectives from European Research. Fraunhofer IRB Verlag (2007) 
2. Lillehagen, F., Karlsen, D.: Visual Extended Enterprise Engineering embedding Knowledge Management, Systems Engineering and Work Execution. In: IEMC 1999 IFIP International Enterprise Modelling Conference, Verdal, Norway (1999)

3. Krogstie, J., Jørgensen, H.D.: Interactive Models for Supporting Networked Organizations. In: Persson, A., Stirna, J. (eds.) CAiSE 2004, LNCS, vol. 3084, pp. 550-563. Springer, Heidelberg (2004)

4. Jørgensen, H.D., Krogstie, J.: Active Models for Dynamic Networked Organisations. In: Dittrich, K.R., Geppert, A., Norrie, M.C. (eds.) CAiSE 2001, LNCS, vol. 2068. Springer, Heidelberg (2001)

5. Lillehagen, F.: The Foundations of AKM Technology. In: Proceedings 10th International Conference on Concurrent Engineering (CE) Conference, Madeira, Portugal (2003)

6. Krogstie, J., Lillehagen, F., Karlsen, D., Ohren, O., Strømseng, K., Thue Lie, F.: Extended Enterprise Methodology. Deliverable 2 in the EXTERNAL project (2000), http: / /research.dnv.com/external/deliverables.html

7. Carstensen, A., et al.: Integrating Requirement and Solution Modelling: Approach and Experiences. In: EMMSAD 2007, Trondheim, Norway (accepted, June 2007)

8. Stirna, J., Persson, A., Sandkuhl, K.: Participative Enterprise Modelling: Experiences and Recommendations. In: Krogstie, J., Opdahl, A.L., Sindre, G. (eds.) CAiSE 2007 and WES 2007. LNCS, vol. 4495, pp. 546-560. Springer, Heidelberg (2007)

9. Vernadat, F.: Enterprise Modelling and Integration: principles and applications. Chapman \& Hall, London (1996)

10. Niemann, K.: From Enterprise Architecture to IT Governance. Vieweg Verlag, Wiesbaden (2006)

11. Fox, M., Gruninger, M.: Enterprise Modelling. AI Magazine 19(3) (1998)

12. Stirna, J., Persson, A.: EKD - An Enterprise Modelling Approach to Support Creativity and Quality in Information Systems and Business Development. In: Halpin, T., Krogstie, J., Proper, E. (eds.) Innovations in Information Systems Modelling: Methods and Best Practices. IGI Publishing (to appear, 2008)

13. Rolland, C., Prakash, N.: Bridging the Gap Between Organisational Needs and ERP Functionality. Requirements Engineering 5, 180-193 (2000)

14. Jacucci, G., Wagner, I., Tellioglu, H.: Design Games as a Part of Social Practice Design: A Case of Employees Elaborating on Organizational Problems. In: Proceedings of 16th European Conference on Information Systems, Galway, Ireland (June 2008)

15. Bubenko, J., Persson, A., Stirna, J.: User Guide of the Knowledge Management Approach Using Enterprise Knowledge Patterns. In: Deliverable D3, project HyperKnowledge (IST2000-28401). Royal Institute of Technology, Sweden 